\title{
PCOS remains a diagnosis of exclusion: a concise review of key endocrinopathies to exclude
}

\author{
Eleni Magdalini Kyritsi ${ }^{1}$, George K. Dimitriadis ${ }^{1,2,3}$, loannis Kyrou ${ }^{1,2,4}$, Gregory \\ Kaltsas $^{1,5}$ \& Harpal S. Randeva ${ }^{1,2,4}$
}

${ }^{1}$ Warwickshire Institute for the Study of Diabetes, Endocrinology and Metabolism, University Hospitals of Coventry and Warwickshire NHS Trust, Coventry CV2 2DX, United Kingdom.

2 Division of Translational and Experimental Medicine, Warwick Medical School, University of Warwick, Coventry CV4 7AL, United Kingdom.

3 Division of Endocrinology and Investigative Medicine, Imperial College London, Hammersmith Campus, London W12 ONN, United Kingdom.

4 Aston Medical Research Institute, Aston Medical School, Aston University, Birmingham, B4 7ET, United Kingdom.

5 Division of Pathophysiology, National and Kapodistrian University of Athens Medical School, Athens 106 79, Greece.

Key words: PCOS, CAH, androgen secreting tumours, hyperprolactinaemia

Address for correspondence:

Professor Harpal S. Randeva

Warwickshire Institute for the Study of Diabetes, Endocrinology and Metabolism, University Hospitals of Coventry and Warwickshire NHS Trust, Coventry CV2 2DX, United Kingdom.

Tel: 02476965972

Fax: 02476965972

E-mail: harpal.randeva@uhcw.nhs.uk

Dr. Eleni Magdalini Kyritsi

Warwickshire Institute for the Study of Diabetes, Endocrinology and Metabolism, University Hospitals of Coventry and Warwickshire NHS Trust, Coventry CV2 2DX, United Kingdom.

Tel: 02476966115

Fax: 02476965972

Email address: Elena.kyritsi@uhcw.nhs.uk 


\section{Summary}

Polycystic ovarian syndrome (PCOS) is a heterogenous disorder associated with clinical, endocrine and ultrasonographic features that can also be encountered in a number of other diseases. It has traditionally been suggested that prolactin excess, enzymatic steroidogenic abnormalities and thyroid disorders need to be excluded before a diagnosis of PCOS is made. However, there is paucity of data regarding the prevalence of PCOS-phenotype in some of these disorders, whereas other endocrine diseases that exhibit PCOS-like features may elude diagnosis and proper management if not considered. The present article reviews the data of currently included entities that exhibit a PCOS-phenotype and those that potentially need to be looked for, and attempts to identify specific features that distinguish them from idiopathic PCOS. 


\section{Introduction}

Clinical and/or biochemical hyperandrogenism and menstrual irregularity are the most common complaints for which premenopausal women seek endocrine consultations ${ }^{1}$. The great majority of such women will be found to have Polycystic Ovarian Syndrome (PCOS) that is associated with a distinct ultrasonographic polycystic ovarian (PCO) appearance ${ }^{1,2}$. Although the aetiology of PCOS has not been delineated, it is considered to represent a functional hyperandrogenic state of ovarian and/or adrenal origin with or without associated insulin resistance ${ }^{2}$. However, the clinical, endocrine and morphological characteristics of PCOS can also be encountered in a number of other conditions of specific etiology $\mathrm{y}^{3-6}$. It has therefore been suggested that the diagnosis of PCOS should be made when other conditions that could present with a similar clinical/biochemical/morphological phenotype, namely thyroid disorders, states of prolactin excess and congenital adrenal hyperplasia, are excluded ${ }^{4,5,6}$ (Table 1). However, the existence of precise indications and extent of investigations that needs to be undertaken to exclude the presence of such disorders, along with others that may also exert a similar phenotype, have not been clearly defined ${ }^{3}$. In contrast to PCOS, some of these latter conditions can be life threatening or associated with increased morbidity if left undiagnosed ${ }^{3}$. It is therefore important to be aware of all these disorders and recognize any particular features that distinguish them from idiopathic PCOS.

\section{Steroidogenic pathway and cortisol biosynthesis deficiencies}

Although the manifestations of classic (complete enzymatic defect) congenital adrenal hyperplasia (CAH) are evident from birth, the non-classic (mild enzymatic defect) form becomes apparent in adolescence or later (late-onset $\mathrm{CAH})^{7}$. The prevalence of late-onset $\mathrm{CAH}$ ranges between 1-10\%, depending on ethnicity, with late onset 21-hydroxylase $(\mathrm{OH})$ deficiency being the most common cause (1:500-1000 in various Caucasian populations) ${ }^{7}$. In cases of CAH, ACTH levels increase to overcome the block in cortisol biosynthesis leading to increased androgen secretion and the development of symptoms/signs similar to those of patients with $\mathrm{PCOS}^{7,8}$. Early initial studies have shown that ultrasonographically detected PCO were found in $83 \%$ of adult patients, $40 \%$ of post-pubertal and $3 \%$ of pre- and peripubertal girls with late-onset $\mathrm{CAH}^{9}$. In view of these findings it was suggested that measurement of early follicular 17-hydroxyprogesterone (17OH-PG) levels, followed by an ACTH-stimulation test in cases with borderline values, is required to distinguish between these two conditions ${ }^{8}$. In an attempt to identify clinical and other features that distinguish PCOS from late onset-CAH subsequent comparative studies showed that patients with $\mathrm{CAH}$ were less likely to demonstrate anovulation than patients with $\mathrm{PCOS}^{10}$. In addition, PCO were found in only $24 \%$ of CAH compared to approximately $90 \%$ of patients with PCOS, whereas maximum ovarian volume and follicle counts, albeit increased in both groups 
compared to controls, were significantly higher in PCOS patients (10.2 vs. $6.1 \mathrm{ml}$ and $11.7 \mathrm{vs}$. 8.1 , respectively $)^{10}$. As expected $170 \mathrm{H}-\mathrm{PG}$ levels were increased in the great majority of $\mathrm{CAH}$ patients, whereas an LH:FSH ratio of $>2$ was found mostly in patients with PCOS. However, besides the presence of some features that may help distinguishing these two conditions, the gold standard in cases of doubt remains the 17-OH-PG response to ACTH stimulation ${ }^{8,9}$. Moreover, urine steroid metabolomics (Gas chromatography/mass spectrometry-GC/MS) has been shown to be a novel, non-invasive, highly sensitive, and specific biomarker tool for the diagnosis of defects in steroidogenesis ${ }^{7}$. Pregnanetriol, 17-OH pregnanolone and pregnanetriolone are considered to be the urinary metabolites typically increased in cases of 21-OH deficiency?. Patients with late-onset $\mathrm{CAH}$ also exhibit metabolic abnormalities and insulin resistance (IR) similar to lean PCOS further enhancing adrenal and ovarian androgen production $^{3,8}$. Although symptomatic treatment of these two conditions may be similar including the administration of anti-androgens and contraceptives, application of etiological treatment in $\mathrm{CAH}$ patients may require the administration of glucocorticoids ${ }^{8,9}$.

\section{States of prolactin excess}

Approximately $20 \%$ of women with PCOS have elevated prolactin levels suggesting that hyperprolactinaemia may reflect an abnormal ovarian response to an altered gonadotrophin drive $^{11}$. In addition, a number of mainly retrospective studies have revealed a $56 \%$ and 50 $67 \%$ prevalence of hirsutism and PCO in hyperprolactinaemic patients, respectively, whereas testosterone levels were found to be higher in hyperprolactinaemic patients with PCO compared to those without $\mathrm{PCO}^{11,12}$. Previous studies have attempted to characterize such patients according to their hormonal response to TRH, GnRH, and metoclopramide, suggesting that hyperprolactinaemia in patients with PCOS may be the result of the abnormal hormonal milieu with or without associated dopamine deficiency leading to anovulatory cycles ${ }^{\mathbf{1 2}}$. As prolactin receptor expression has been documented in the adrenals, it has also been suggested that hyperprolactinaemia could augment adrenal androgen secretion ${ }^{3,13}$. However, there is a lack of well conducted prospective studies evaluating the prevalence of PCO/PCOS and identification of possible underlying mechanisms in women with different causes of hyperprolactinaemia, and thus the association between prolactin excess and PCOS might not be causal, but rather an integral part of PCOS.

\section{Thyroid disorders}

Increase in ovarian volume and presence of ovarian cystic changes have been reported in patients with primary hypothyroidism $(\mathrm{PH})$, whereas thyroid disorders and autoimmunity are more common in women with $\mathrm{PCOS}^{14}$. Although the underlying mechanism has not 
been delineated it is hypothesized that increased prolactin secretion, that accompanies $\mathrm{PH}$, affects gonadotrophin secretory pattern and promotes adrenal androgen secretion ${ }^{14}$. In addition, increased TSH levels may increase collagen deposition and overall ovarian volume. In a study of 26 patients with PH and mean TSH levels of $57.1 \mathrm{mU} / \mathrm{L}$, ovarian volumes were larger than controls and a number of patients acquired multiple ovarian cysts that all reverted to normal following thyroxine replacement therapy ${ }^{15}$. In addition, decreased sex hormone binding globulin (SHBG) and increased free testosterone levels were found in hypothyroid patients, whereas increased SHBG and dehydroepiandrosterone sulfate (DHEAS) levels have been described in hyperthyroid patients ${ }^{16}$. However, there are no convincing data derived from large scale studies demonstrating a direct relation of thyroid dysfunction or association of thyroid autoimmunity with PCOS.

More recent evidence suggests that a number of other conditions may also present with a PCOS-phenotype. It is therefore important to familiarize with the presence of such disorders as early presentation may not always be typical of overt disease raising clinical/endocrinological suspicion.

\section{Cushing's syndrome (CS)}

A significant number of patients with CS develop hirsutism and approximately $70-80 \%$ menstrual abnormalities, besides the absence of raised androgen levels ${ }^{17,18}$. As all patients with CS have low SHBG levels, as a result of cortisol induced IR and hyperinsulinemia, the free androgen index is increased leading to hirsutism ${ }^{18}$. PCO are found in $46 \%$ of patients with CS; in addition, there is a strong negative correlation between menstrual pattern and gonadotrophins with cortisol levels ${ }^{17}$. Thus, it has been suggested that when cortisol levels are moderately raised, gonadotrophin stimulation to the ovaries is maintained leading to an estrogen sufficient PCOS-like phenotype, whereas highly elevated cortisol levels lead to a hypogonadotropic hypogonadism state ${ }^{17,3}$. In such cases, hyperinsulinemia may act as a cogonadotropin stimulating the ovaries, thus maintaining ovarian steroid output and leading to the development of $\mathrm{PCO}^{17,3}$. This hypothesis is supported further by reports of patients with early or mild CS being misdiagnosed as $\mathrm{PCOS}^{19}$.

\section{Acromegaly}

Between $40-80 \%$ of patients with acromegaly develop hirsutism and menstrual irregularity ${ }^{20}$. The majority of patients with large pituitary tumours are associated with complete or partial gonadotrophin deficiency, with or without concomitant 
hyperprolactinemia, leading to a hypogonadotrophic hypogonadal state ${ }^{20}$. However, acromegalic patients presenting with a PCOS-like phenotype exhibiting an exaggerated LH response to $\mathrm{LHRH}$ have also been described ${ }^{20,3}$. Such patients usually have pituitary microadenomas and maintain an estrogen sufficient milieu ${ }^{20,3}$. Although the majority of patients with acromegaly do not have high androgen levels, they exhibit low SHBG levels that correlate inversely with GH levels, a finding that could explain the presence of hirsutism despite normal androgen levels ${ }^{20}$. A recent prospective study evaluating 14 pre-menopausal patients with active acromegaly showed that $50 \%$ had PCO and $45 \%$ fulfilled the criteria of $\mathrm{PCOS}^{21}$. Women with PCOS had increased mean ovarian volumes and characteristic morphology compared to those without, higher IGF-1 and testosterone levels and lower SHBG levels ${ }^{21}$. It is prudent to suggest that GH/IGF-1 excess or IR can affect steroid production, SHBG levels and ovarian function, and induce a PCOS-phenotype even in a gonadotrophin deficient state ${ }^{21}$.

\section{Adrenal and ovarian androgen secreting tumours}

Androgen-secreting neoplasms, mostly arising from the adrenals or ovaries, are relatively rare and potentially life-threatening and may be associated with virilization (clitoromegaly, deepening of the voice, frontal balding and muscle hypertrophy) (Table 2 2 $^{22}$. It is suggested that such tumours could be identified based on clinical (rapid onset of symptoms and virilization), and biochemical features (extremely high androgen levels), whilst cases of slowly evolving tumours exhibiting a wide range of elevated androgen levels can occur $3,23,24$. It has been suggested that only testosterone levels $>5 \mathrm{nmol} / \mathrm{L}$ are highly suggestive of androgen-secreting tumours ${ }^{23,24}$. The most common virilising ovarian tumour is arrhenoblastoma, which can occasionally be gonadotrophin dependent ${ }^{3,22}$. Adrenal androgen producing tumours are usually malignant, although benign tumours have also been reported $3,22,25$. Malignant adrenal tumours characteristically produce androgens (mainly testosterone and DHEA-S) and/or cortisol resulting in a mixed phenotype; however, approximately $10 \%$ can present solely with virilization ${ }^{3,22}$. Prompt diagnosis and treatment of these tumours, particularly adrenal carcinomas, is important as disease stage at diagnosis correlates with survival ${ }^{3,22}$.

The use of some medications may be associated with hyperandrogenism and menstrual irregularity. Although exogenous androgen administration was thought to induce PCO/PCOS, this has been proven only when supra-physiological doses are given (as in cases of female-to-male transsexuals) ${ }^{26}$. Valproic acid was initially considered to induce PCOS, but this was not substantiated by further studies, whereas drugs producing hirsutism have not been shown to induce PCO/PCOS 3 . 


\section{Other rarer causes exhibiting a PCOS-phenotype}

Relatively rare disorders that exhibit distinctive clinical and/or endocrinological features can also present with a PCOS-like phenotype. In the HAIR-AN syndrome [hyperandrogenism $(H A), I R$ and acanthosis nigricans (AN)] the degree of IR and subsequent hyperinsulinemia lead to increased ovarian androgen production ${ }^{27}$. Such patients usually present from puberty and have variable levels of hyperandrogenism ${ }^{27}$. In addition, in a number of common disorders associated with hyperinsulinemia and IR, such as type 2 diabetes mellitus, gestational diabetes and obesity, the prevalence of PCO/PCOS is substantially increased, but this is anticipated due to the underlying disorder ${ }^{3}$.

Glucocorticoid resistance (GR) or altered cortisol metabolism states can also lead to the development of a PCOS-phenotype albeit being extremely rare ${ }^{28,29}$. In the former, compensatory ACTH rise leads to excessive adrenal androgen production and development of hirsutism, menstrual irregularity and infertility mimicking $\mathrm{PCOS}^{28}$. Elevated cortisol and ACTH levels that maintain normal circadian rhythm and absence of a CS-phenotype are the hallmarks for making a proper diagnosis ${ }^{28}$. Enzymatic defects, such as overactivity of $5 \alpha-$ reductase that converts testosterone to the more potent dihydro-testosterone, as well as deficiency of 11-oxoreductase that converts cortisone to cortisol, are associated with enhanced cortisol metabolism, ACTH hypersecretion and subsequent hyperandrogenism. However, both these conditions are extremely rare causes of PCOS ${ }^{3,29}$.

\section{Conclusions}

Most women with hyperandrogenism and chronic anovulation will be found to have PCOS. However, as these findings are not specific to PCOS the number of disorders that needs to be excluded should be expanded. Early onset and low grade states of cortisol or GH excess along with evolving androgen secreting tumours should be considered and promptly investigated in the presence of relevant clinical/endocrine settings. Conditions associated with severe insulin resistance also need to be considered as they can frequently be associated with a PCOS-phenotype (Figure 1). 


\section{References}

1. Balen, A., Rajkowha, M. (2003) Polycystic ovary syndrome--a systemic disorder? Best Practice \& Research Clinical Obstetrics \& Gynaecology, 17, 263-274.

2. Franks, S. (1995) Polycystic ovary syndrome. The New England Journal of Medicine, 333, 853-861.

3. Kaltsas, G.A., Isidori, A.M., Besser, G.M. et al (2004) Secondary forms of polycystic ovary syndrome. Trends in Endocrinology \& Metabolism, 15, 204-210.

4. Carmina, E. (2004) Diagnosis of polycystic ovary syndrome: from NIH criteria to ESHREASRM guidelines. Minerva ginecologica, 56, 1-6.

5. Rotterdam ESHRE/ASRM-Sponsored PCOS consensus workshop group (2004) Revised 2003 consensus on diagnostic criteria and long-term health risks related to polycystic ovary syndrome (PCOS). Human Reproduction, 19, 41-47.

6. Azziz, R., Carmina, E., Dewailly, D. et al (2006) Positions statement: criteria for defining polycystic ovary syndrome as a predominantly hyperandrogenic syndrome: an Androgen Excess Society guideline. The Journal of Clinical Endocrinology and Metabolism, 91, 42374245.

7. Krone, N., Dhir, V., Ivison, H.E. et al (2007) Congenital adrenal hyperplasia and P450 oxidoreductase deficiency. Clinical Endocrinology, 66, 162-172.

8. Moran, C., Azziz, R. (2003) 21-hydroxylase-deficient nonclassic adrenal hyperplasia: the great pretender. Seminars in Reproductive Medicine, 21, 295-300.

9. Hague, W.M., Adams, J., Rodda, C. et al (1990) The prevalence of polycystic ovaries in patients with congenital adrenal hyperplasia and their close relatives. Clinical Endocrinology, 33, 501-510.

10. Pall, M., Azziz, R., Beires, J. et al (2010) The phenotype of hirsute women: a comparison of polycystic ovary syndrome and 21-hydroxylase-deficient nonclassic adrenal hyperplasia. Fertility and Sterility, 94, 684-689.

11. Abdel Gadir, A., Khatim, M.S., Mowafi, R.S. et al (1992) Implications of ultrasonically diagnosed polycystic ovaries. I. Correlations with basal hormonal profiles. Human Reproduction, 7, 453-457.

12. Işik, A.Z., Gülekli, B., Zorlu, C.G. et al (1997) Endocrinological and clinical analysis of hyperprolactinemic patients with and without ultrasonically diagnosed polycystic ovarian changes. Gynecologic and Obstetric Investigation, 43, 183-185. 
13. Paoletti, A.M., Cagnacci, A., Soldani, R. et al (1995) Evidence that an altered prolactin release is consequent to abnormal ovarian activity in polycystic ovary syndrome. Fertility and Sterility, 64, 1094-1098.

14. Benetti-Pinto, C.L., Berini Piccolo, V.R., Garmes, H.M. et al (2013) Subclinical hypothyroidism in young women with polycystic ovary syndrome: an analysis of clinical, hormonal, and metabolic parameters. Fertility and Sterility, 99, 588-592.

15. Muderris, I.I., Boztosun, A., Oner, G. et al (2011) Effect of thyroid hormone replacement therapy on ovarian volume and androgen hormones in patients with untreated primary hypothyroidism. Annals of Saudi Medicine, 31, 145-151.

16. Tagawa, N., Tamanaka, J., Fujinami, A. et al (2000) Serum dehydroepiandrosterone, dehydroepiandrosterone sulfate, and pregnenolone sulfate concentrations in patients with hyperthyroidism and hypothyroidism. Clinical Chemistry, 46, 523-528.

17. Kaltsas, G.A., Korbonits, M., Isidori, A.M. et al (2000) How common are polycystic ovaries and the polycystic ovarian syndrome in women with Cushing's syndrome? Clinical Endocrinology, 53, 493-500.

18. Lado-Abeal, J., Rodriguez-Arnao, J., Newell-Price, J.D. et al (1998) Menstrual abnormalities in women with Cushing's disease are correlated with hypercortisolemia rather than raised circulating androgen levels. The Journal of Clinical Endocrinology and Metabolism, 83, 3083-3088.

19. Brzana, J., Yedinak, C.G., Hameed, N. et al (2014) Polycystic ovarian syndrome and Cushing's syndrome: a persistent diagnostic quandary. European Journal of Obstetrics \& Gynecology and Reproductive Biology, 175, 145-148.

20. Kaltsas, G.A., Mukherjee, J.J., Jenkins, P.J. et al (1999) Menstrual irregularity in women with acromegaly. The Journal of Clinical Endocrinology and Metabolism, 84, 2731-2735.

21. Kaltsas, G.A., Androulakis, I.I., Tziveriotis, K. et al (2007) Polycystic ovaries and the polycystic ovary syndrome phenotype in women with active acromegaly. Clinical Endocrinology, 67, 917-922.

22. Markopoulos, M.C., Kassi, E., Alexandraki, K.I. et al (2015) Hyperandrogenism after menopause. European Journal of Endocrinology, 172, R79-91.

23. Kaltsas, G.A., Isidori, A.M., Kola, B.P. et al (2003) The value of the low-dose dexamethasone suppression test in the differential diagnosis of hyperandrogenism in women. The Journal of Clinical Endocrinology and Metabolism, 88, 2634-2643. 
24. Derksen, J., Nagesser, S.K., Meinders, A.E. et al (1994) Identification of virilizing adrenal tumors in hirsute women. The New England Journal of Medicine, 331, 968-973.

25. Cordera, F., Grant, C., van Heerden, J. et al (2003) Androgen-secreting adrenal tumors. Surgery, 134, 874-880.

26. Moore, E., Wisniewski, A., Dobs, A. (2003) Endocrine treatment of transsexual people: a review of treatment regimens, outcomes, and adverse effects. The Journal of Clinical Endocrinology and Metabolism, 88, 3467-3473.

27. Barbieri, R.L., Ryan, K.J. (1983) Hyperandrogenism, insulin resistance, and acanthosis nigricans syndrome: a common endocrinopathy with distinct pathophysiologic features. American Journal of Obstetrics and Gynecology, 147, 90-101.

28. Charmandari, E., Kino, T., Ichijo, T. et al (2008) Generalized glucocorticoid resistance: clinical aspects, molecular mechanisms, and implications of a rare genetic disorder. The Journal of Clinical Endocrinology and Metabolism, 93, 1563-1572.

29. Stewart, P.M., Shackleton, C.H., Beastall, G.H. et al (1990) 5 alpha-reductase activity in polycystic ovary syndrome. Lancet, 335, 431-433.

30. Piaditis, G., Angellou, A., Kontogeorgos, G. et al (2005) Ectopic bioactive luteinizing hormone secretion by a pancreatic endocrine tumor, manifested as luteinized granulosathecal cell tumor of the ovaries. The Journal of Clinical Endocrinology and Metabolism, 90, 2097-2103. 


\section{Legends}

Table 1. Current criteria for diagnosing PCOS

Table 2. Features of tumours associated with hyperandrogenemia

Figure 1ף. Diagnostic algorithm for the identification of PCOS mimicking conditions. 
Table 1. Current criteria for diagnosing PCOS

\begin{tabular}{|c|c|c|c|c|}
\hline & & $\begin{array}{c}\text { NIH } \\
\text { criteria }\end{array}$ & $\begin{array}{l}\text { Rotterdam } \\
\text { criteria }\end{array}$ & $\begin{array}{c}\text { Androgen } \\
\text { excess Society } \\
\text { criteria }\end{array}$ \\
\hline Hyperandrogenism & $\begin{array}{l}\text { Clinical: hirsutism, acne, androgenic alopecia } \\
\text { Biochemical: increased total, bioavailable or } \\
\text { free testosterone levels }\end{array}$ & + & + & + \\
\hline Oligo/anovulation & $\begin{array}{l}\text { Bleeding intervals of }<21 \text { (polymenorrhea) or }> \\
35 \text { days (oligo- or amenorrhea) }\end{array}$ & + & + & + \\
\hline $\begin{array}{l}\text { Exclusion of other } \\
\text { diagnoses }\end{array}$ & $\begin{array}{l}\text { Thyroid disease } \\
\text { Non-classic congenital adrenal hyperplasia } \\
\text { Hyperprolactinaemia }\end{array}$ & + & + & + \\
\hline PCO morphology & $\begin{array}{l}12 \text { or more } 2-9 \mathrm{~mm} \text { in diameter follicles and/or } \\
\text { ovarian volume }>10 \mathrm{ml} \text { in either ovary }\end{array}$ & & + & + \\
\hline
\end{tabular}

All criteria require exclusion of other diagnoses with a PCO-like phenotype. Rotterdam require any 2 of remaining 3 criteria, Androgen Excess Society requires hyperandrogenism with 1 of the remaining 2 criteria, whereas NIH do not include PCO morphology. (PCO= polycystic ovarian) 
Table 2: Features of tumours associated with hyperandrogenemia

\begin{tabular}{|c|c|c|c|c|c|c|}
\hline Tumour & $\begin{array}{l}\text { Age at presentation } \\
\text { (range, years) }\end{array}$ & $\begin{array}{l}\text { Incidence in relevant } \\
\text { tumour entity (\%) }\end{array}$ & Hormone secretion & Symptoms & Bilaterality & Malignant potential \\
\hline $\begin{array}{l}\text { Adrenal tumours } \\
\text { Adrenal carcinoma } \\
\text { Adrenal adenoma }\end{array}$ & $\begin{array}{c}\text { 3-79 } \\
\text { Variable }\end{array}$ & $\begin{array}{l}4-5 \% \\
<1 \%\end{array}$ & $\begin{array}{l}\text { Testosterone, DHEA-S, } \\
\text { Androstenedione }\end{array}$ & $\begin{array}{c}\text { CS } \\
\text { Virilization }\end{array}$ & Unilateral & $\begin{array}{c}\text { High } \\
\text { Very low }\end{array}$ \\
\hline \multicolumn{7}{|l|}{ Ovarian tumours } \\
\hline Androblastoma (Sertoli-Leydig cell tumours) & $2-75$ & 0.5 & Androgen, rarely estrogens & Virilization $1 / 3$ & $1-2 \%$ & Low \\
\hline Granulosa cell tumours & $40-70$ & $2-3$ & Estrogens, rarely androgens & Rarely virilization & $5 \%$ & Low \\
\hline Sertoli cell tumours & 7-79 & 0.1 & Androgens rarely estrogens & Virilization in $30 \%$ & $1-2 \%$ & Low \\
\hline Hillus cell tumour & $6^{\text {th }}$ decade & 0.02 & Androgens & $\begin{array}{l}\text { Hirsutism and virilization 50- } \\
75 \%\end{array}$ & Rare & Very rare \\
\hline \multicolumn{7}{|l|}{ Other type of tumours } \\
\hline NET (pNET) tumour ${ }^{30}$ & Can be variable & $<0.1 \%$ & LH & $\begin{array}{l}\text { Hirsutism, menstrual } \\
\text { irregularity }\end{array}$ & $\begin{array}{l}\text { Extra- } \\
\text { adrenal }\end{array}$ & Intermediate \\
\hline
\end{tabular}

DHEA-S= dehydroepiandrosterone sulfate; $C S=$ Cushing's syndrome; NET= neuroendocrine tumour; pNET= pancreatic neuroendocrine tumour 


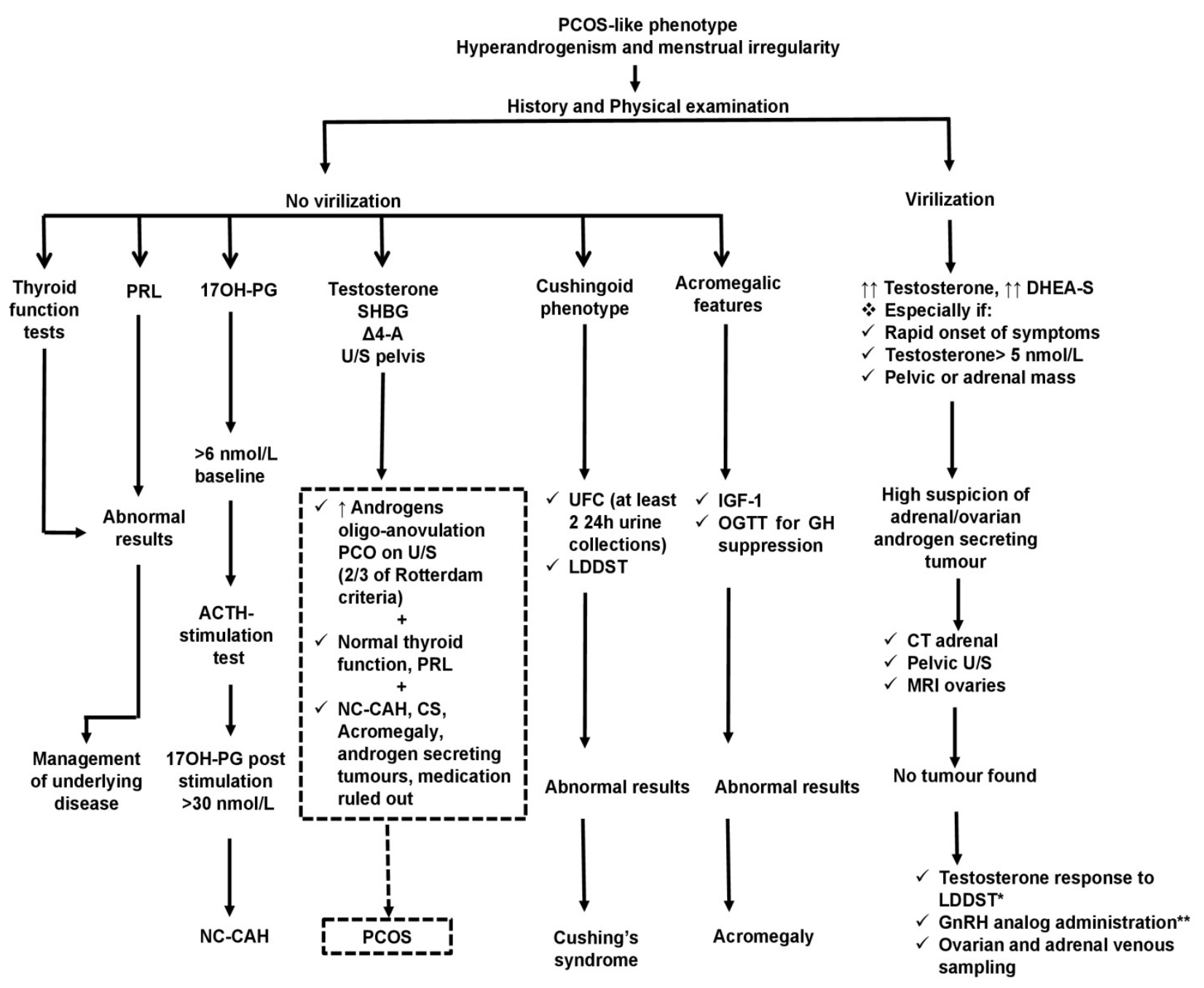

Figure 1ๆ. Diagnostic algorithm for the identification of PCOS mimicking conditions.

17OH-PG = 17-hydroxyprogesterone; $\Delta 4-\mathrm{A}=\Delta 4$-androstenedione; DHEA-S= dehydroepiandrosterone sulfate; $\mathrm{U} / \mathrm{S}=$ ultrasound; UFC= urinary free cortisol; LDDST = Low-dose dexamethasone suppression test $(0.5 \mathrm{mg}$ every $6 \mathrm{~h}$ for $48 \mathrm{~h}$ ); OGTT= oral glucose tolerance test ( 75 gr glucose per os) ; NC-CAH = nonclassic (late-onset) congenital adrenal hypeplasia; $\mathrm{CS}=$ Cushing's syndrome; $\mathrm{PCO}=$ polycystic ovaries; $\mathrm{PCOS}=$ polycystic ovarian syndrome; $\mathrm{CT}=$ computed tomography; $\mathrm{MRI}=$ magnetic resonance imaging.

*Testosterone response to LDDST is defined as $40 \%$ decrease or normalization of serum testosterone following LDDST.

**GnRH analog administration has been shown to suppress the gonadotrophins leading to normalization of elevated testosterone levels in patients with ovarian androgen-secreting tumours, as well as hyperthecosis, although it cannot assist in the differential diagnosis between the two conditions.

9) Amended from Reference 22 